\title{
COMMEMORATING THE PAST AND LOOKING TOWARDS THE FUTURE
}
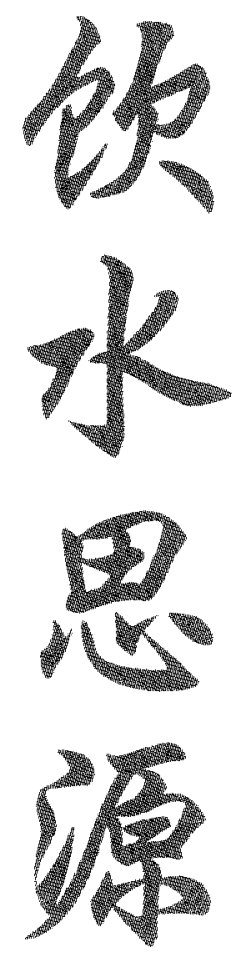
This page is intentionally left blank 


\section{第三届全球华人物理学大会 \\ OCPA 2000: Proceedings of the Third Joint Meeting of Chinese Physicists Worldwide \\ COMMEMORATING THE PAST AND LOOKING TOWARDS THE FUTURE

\author{
31 July - 4 August, 2000 \\ The Chinese University of Hong Kong, HK \\ Jointly organized by \\ Overseas Chinese Physics Association \\ The Chinese University of Hong Kong
}

Editors

Ngee-Pong Chang

City College of CUNY, USA

Kenneth Young

Chinese University of Hong Kong, HK

Hon Ming Lai

Chinese University of Hong Kong, HK

Cheuk-Yin Wong

Oak Ridge National Laboratory, USA 
Published by

World Scientific Publishing Co. Pte. Ltd.

P O Box 128, Farrer Road, Singapore 912805

USA office: Suite 1B, 1060 Main Street, River Edge, NJ 07661

UK office: 57 Shelton Street, Covent Garden, London WC2H 9HE

\section{British Library Cataloguing-in-Publication Data}

A catalogue record for this book is available from the British Library.

\section{COMMEMORATING THE PAST AND LOOKING TOWARDS THE FUTURE OCPA 2000 \\ Proceedings of the Third Joint Meeting of Chinese Physicists Worldwide \\ Copyright $\odot 2002$ by World Scientific Publishing Co. Pte. Ltd.}

All rights reserved. This book, or parts thereof, may not be reproduced in any form or by any means, electronic or mechanical, including photocopying, recording or any information storage and retrieval system now known or to be invented, without written permission from the Publisher.

For photocopying of material in this volume, please pay a copying fee through the Copyright Clearance Center, Inc., 222 Rosewood Drive, Danvers, MA 01923, USA. In this case permission to photocopy is not required from the publisher.

ISBN $\quad 981-238-122-8$ 


\author{
Co-sponsored by \\ Physical Society of Hong Kong \\ Southeast Asian Theoretical Physics Association \\ City University of Hong Kong \\ Hong Kong Baptist University \\ Hong Kong Polytechnic University \\ Hong Kong University of Science and Technology \\ University of Hong Kong
}

\title{
Supported by
}

Croucher Foundation

K. C. Wong Education Foundation

Hsin Chong - K. N. Godfrey Yeh Education Fund

Lee Hysan Foundation

Lee Foundation (Singapore)

C. S. Wu Foundation

Mr. William W. P. Zen 


\section{International Advisory Committee}

Leroy Chang, Hong Kong U of Sci \& Tech

Jia-Er Chen, Peking U

S. P. Chia, U of Malaya

Alfred Cho, AT\&T

Paul C. W. Chu, U of Houston

Steven Chu, Stanford U

Duan Feng, Nanjing U

Patrick Lee, MIT

T. D. Lee, Columbia U

Yuan T. Lee, Academia Sinica (Taipei)

C. C. Lin, MIT

K. Y. Lo, Inst Astrophys, AS (Taipei)

K. K. Phua, World Scientific
Yuen-Ron Shen, UC Berkeley

S.C.C. Ting, MIT

Tien T. Tsong, Inst Phys, AS (Taipei)

Dan Tsui, Princeton

Xi-De Xie, Fudan U

Nai-Yan Wang, Natural Science Foundation China

M. K. Wu, N. Tsinghua U, Hsinchu

T. Y. Wu, Academia Sinica (Taipei)

C. N. Yang, SUNY Stony Brook \& Chinese U of HK

S. T. Yau, Harvard U \& Chinese U of HK

Zhong-Xian Zhao, Inst Phys, AS (Beijing)

Guang-Zhao Zhou, Academia Sinica (Beijing)

Guang-Ya Zhu, Chinese Assn of Sci \& Tech

\section{International Organizing Committee}

Co-chair: Ngee-Pong Chang, City College of CUNY Co-chair: Kenneth Young, CUHK

Conference Secretary: Ling-Fong Li, Camegie Mellon U

Hong-Mo Chan, Rutherford Lab

Moses Chan, Penn State U

Darwin Chang, Tsinghua U, Hsinchu

Lay-Nam Chang, Virginia Tech

Tu-Nan Chang, USC

Alex Chao, SLAC

Liu Chen, UC Irvine

Pisin Chen, SLAC

Yang-Fang Chen, Taiwan U

Hai-Yang Cheng, Inst Phys, AS (Taipei)

John Cheng-Chung Chi, Taiwan

Raymond Chiao, UC Berkeley

Chih-Yung Chien, Johns Hopkins U

C. L. Choy, Polytech U HK

Shou-Xian Fang, Inst HE Phys, AS (Beijing)

Da-Hsuan Feng, Drexel U

Sun-Yiu Fung, UC Riverside

Zi-Zhao Gan, Peking U

Jason Ho, Ohio State U

Bambi Hu, Baptist U of HK

Zhong-Yi Hua, Fudan U

Pauchy Hwang, Taiwan U, Taipei

Tao Huang, IHEP CAS (Beijing)

Chi-Sing Lam, McGill U

Leo Woon-Ming Lau, Chinese U HK

Dung-Hai Lee, UC Berkeley

Shih-Chang Lee, Inst Physics, AS (Taipei)
S. T. Lee, City U HK

Hua-Zhong $\mathrm{Li}$, Zhongshan $\mathrm{U}$

Keh-Fei Liu, Kentucky U

Cheuk-Yiu Ng, lowa State U

Hwa-Tung Nieh, Tsinghua U, Beijing

Zhong-Can Ou-Yang, ITP, AS (Beijing)

Jen-Chieh Peng, LANL

Lu Sham, UC San Diego

Zhi-Xun Shen, Stanford U

Ping Sheng, Hong Kong U of Sci \& Tech

Zhao-Bin Su, Inst of Theo Phys, AS (Beijing)

Wai-Mo Suen, U Washington St. Louis

Shuh Y. Tong, Hong Kong U

Wu-Ki Tung, MSU

En-Ge Wang, Inst of Physics, AS (Beijing)

Cheuk-Yin Wong, ORNL

Ding-Chang Xian, IHEP, Beijing

Guo-Zhen Yang, Inst of Physics, AS (Beijing)

Tung-Mow Yan, Cornell U

Yeong-Der Yao, Inst Physics, AS (Taipei)

Nai-Chang Yeh, Cal Tech

Bing-Lin Young, Iowa State

Chi Yuan, Inst Astrophysics, AS (Taipei)

Shou-Cheng Zhang, Stanford U

Kai-Hua Zhao, Peking U

Zhi-Peng Zheng, Inst H.E. Phys, AS (Beijing)

\section{Local Organizing Committee}

Chair: Hon-Ming Lai (CUHK)

Secretary: Hai-Qing Lin (CUHK)

Ho-Fai Cheung (City U)

Ming-Chung Chu (CUHK)

Chi-Hang Lam (PolyU)

Leo Woon-Ming Lau (CUHK)

Yuk-Yan Lee (CUHK)

Tai-Kai Ng (USTHK)
Treasurer: Kin-Wah Yu (CUHK)

PSHK Representative: Kwong-Sang Cheng (HKU)

$\mathrm{Zi}$-Dan Wang (HKU)

Wing-Hung Wong (CUHK)

Ke-Qing Xia (CUHK)

John Kwok-Fung Yau (CUHK)

Shi-Yao Zhu (Baptist U) 


\section{Contents}

Opening Address

N.-P. Chang

Plenary Sessions

T. Y. Wu Memorial Session

C. N. Yang

Introduction

K. Huang

My Respected Teacher Prof. Ta-You Wu

C. S. Shen

The Later Years of Prof. Ta-You Wu

T. D. Lee

A New Method to Solve N-Dimensional Quantum Wave Functions

C. S. Wu Memorial Session

C.-H. Liu

Wu Chien-Shiung the First Lady of Physics

Y.-H. Chu

High-Energy Astrophysics at the Low-Energy End

N.-C. Yeh, C.-T. Chen, C.-C. Fu, Z. Huang, T. A. Corcovilos,

R. P. Vasquez and D. M. Strayer

Recent Development in the Science and Technology of Superconductivity

Xie Xi-De Memorial Session

X. Wang

Life and Times of Prof. Xie Xi-De

Other Plenary Sessions

G. $\mathrm{Ai}$

On the Space Solar Telescope in China

P. Chen

Quantum Aspects of Beam Physics 
J. H. Hou, J. Yang, Z. Li and Q. Zhu

Physics and Chemistry with Single Molecule: Experiment and Theory

H. Zhou, Y. Zhang and Z.-C. Ou-Yang

The Mechanical Aspects of Double- and Single-Stranded DNA Chains

\section{Condensed Matter Physics}

D. Chen

Imaging Electron Fringes on a Quantum Wedge

M.-K. Chen

The Relativistic Effects of Doubly Excited ${ }^{1,3} \mathrm{P}^{\circ}$ Resonances in $\mathrm{H}^{-}$between $\mathrm{n}=2$ and $3 \mathrm{H}$ Thresholds

H.-J. Gao

From "Seahorse" to "Molecular Recording"

C. D. Gong, X. J. Chen and H. Q. Lin

Pressure Effect on Critical Temperature of Hole-Doped Cuprates Superconductors

140

W. Kuo, J. H. Shyu, Y. J. Chien, D. S. Chung, M. L. Leung and C. D. Chen Scaling Behavior of Superconductor-Insulator Transition in One-Dimensional Arrays of Small Josephson Junctions

K. M. Leung and C.-H. Lin Modal Transmission-Line Theory of Photonic Band-Gap Structures

J. C. Li and Y. Wang

Neutron Scattering and Its Application in Condensed Matters Physics

H. Q. Lin

Computational Many-Body Physics and Parallel Computation in Hong Kong

M. Ma, A. Joshi and F.-C. Zhang

Theory for Insulating $\mathrm{V}_{2} \mathrm{O}_{3}$

154

H. Mehrez, H. Guo, J. Wang and C. Roland

Conductance of Carbon Nanotubes Acting as Quantum Dots

S.-F. Ren and D. Lu

Quantum Confinement of Phonon Modes in INAS Quantum Dots

J. X. Tang

The Polyelectrolyte Nature and Large Scale Self-Assembly of the Protein Filaments in F-actin

L. Xu, P. Yin and Y. Wang

A Proposal for AC Magnetic Guide and Trap of Cold Atoms 
Y. B. Xu

Large Scale Crystal of $\mathrm{C}_{60}$ and PES Study of the Electronic Band Structure of $\mathrm{K}_{3} \mathrm{C}_{60}$

Yu. Zadorozhny, D. R. Herman and Y. Liu

Magnetic Fingerprints in Superconducting $\mathrm{Au}_{0.7} \operatorname{In}_{0.3}$ Cylinders

H. F. Zhang, W. T. Leung and O. K. C. Tsui

Effect of Chain Ends and Entanglement on Segmental Mobility of Polymers under Confinement

\section{High Energy and Nuclear Physics}

H.-M. Chan

Experimental Implications of the Dual Colour Solution to the Generation Puzzle

N.-P. Chang

Field Theory of the Superluminal Majorana Neutrino and its Oscillation

L3 Beijing Group, G. M. Chen

Study on the Property of $\tau$ Hadronic Decays

S.-Y. Fung

From BEVALAC to AGS

C.-Q. Geng

Rare Kaon Decays

Y.-P. Kuang and Qing Wang

Formal Derivation of the Chiral Lagrangian for Pseudoscalar Mesons from

Quantum Chromodynamics

C.-S. Lam

The Glue Inside a Very Large Nucleus

S. Li, O. Zhang and Z. Zhu

Decomposition of $S U(N)$ Connection and Mass Gap in its Effective Theory

X.-Q. Luo and E. B. Gregory

Recent Progress of Lattice QCD in China

Y. Ma, on behalf of the L3 Collaboration

Cosmic Ray Muon Measurement by L3 Spectrometer at CERN

T. S. Tsou

Yang-Mills Duality as Origin of Generations, Quark Mixing and Neutrino Oscillations

C.-Y. Wong and H. W. Crater

Relativistic Generalization of the Post-Prior Equivalence for Reaction of Composite Particles 
H. T. Wong and J. Li

Scintillation Crystal Detector for Low Energy Neutrino Physics

J. S. Wu, M. R. Strayer and M. Baranger

TDHF Periodic Orbits and Nuclear Collective States

Q. Xie and S. Li

Contribution of Colour-Singlet Process $\Upsilon \rightarrow J / \Psi+c \bar{c} g$ to $\Upsilon \rightarrow J / \Psi+X$ and its

Indication

Y.-P. Yi, Y.-P. Kuang and Q. Wang

Nonperturbative Effects in Operator Product Expansion as the Origin of the

Enhancement Factors in the p-meson QCD Sum Rule

\section{Theoretical Physics and Statistical Mechanics}

B.-Y. Hou and W.-L. Yang

Generalized Yang-Baxter Relations for Lattice Models and Field Theories

G. Hu, M. Zhan and Y. Zhang

Transition from High-Dimensional Chaos to Periodicity by Weak Mutual

Resonant Interactions

J. X. Lu

Why are Perturbative Superstring Theories Incomplete?

Y. J. Ng

Clocks, Computers, Black Holes, Spacetime Foam and Holographic Principle

G. Shiu

Recent Developments in M Physics

C.-I. Tan

AdS/CFT Correspondence for QCD and Pomeron in Strong Coupling Limit

J.-S. Wang

Flat-Histogram and Free Energy Calculation

Z. Xu

Spinor Calculus and its Application to Gauge Theory, Supersymmetry and Gravity

C. J. Zhu

Evidence for Nonvanishing Cosmological Constant in Nonsusy Superstring Models

\section{Astrophysics and Plasma Physics}

Y. P. Chen, M. F. Ye and Q. D. Chen

Equilibrium and Oscillation of Dust Cluster System Attraction Mechanism

Z. G. Dai

Strange Stars and their Astrophysical Implications 
Y. P. Jing

Warm Dark Matter Model of Galaxy Formation

S. Kwok

Chemical Synthesis in the Circumstellar Environment

Y.-Q. Lou

Large-Scale Magnetohydrodynamics of Magnetized Spiral Galaxies

A. T. Y. Lui

The Practical Aspect of Space Weather Research

R. Nan, B. Peng, Y. Qiu, W. Zhu, L. Zhu, Y. Su, X. Ying and F. Li

Preliminary Works on the FAST Project

Y. Tan

YBJ-ARGO Project - A High Altitude Experiment for Cosmic Ray and Astroparticle Physics

X.-P. Wu

The Baryon Fractions in Clusters of Galaxies

J. Yang

Galactic Star Forming Region: Local and Distant

Y.-Y. Zhou

$\mathrm{FeK}_{\alpha}$ Lines in Active Galactic Nuclei

282

Atomic, Molecular, Optical and Chemical Physics

C. G. Bao

What Resonances Can Be Observed during Ps-Ps and Ps-Ps* Collisions

W. Deng, S. Zhao and J. Ouyang

Theoretical Analysis on Four-Wave Mixing with Bose-Einstein Condensates of Dilute Atomic Gases

D.-S. Guo

Atoms in Strong Laser Fields and Nonperturbative QED

W. Kong

Electronic Spectroscopy of Oriented Medium Sized Molecules:

Principle and Applications

K. K. Liang, S. H. Lin, and M. Hayashi

Exciton Model of LH2 Antenna Complex in Photosynthetic Purple Bacteria

D. L. Phillips, K. H. Leung, C.-M. Che, M.-C. Tse, V. M. Miskowski

Resonance Raman Investigation of Metal-Metal Binding Interactions 
Y. Shih

Quantum Teleportation with Complete Set Bell State Measurement

W. Sun, W. Huang, X. P. Xie, Z. P. Zhong, P. Xue and Y. X. Xu

A Study on the Triply Excited Double Rydberg States of La Atom

306

H.-F. Wang

In-Situ Spectroscopic Probe of Solid and Liquid Interfaces in Colloidal Solutions

K. Xu, Z. Zhong and L. Zhu

The Investigations of Absolute Optical Oscillator Strengths and Generalized Oscillator Strengths

Z.-C. Yan

High-Precision Calculations for Four-Body Atomic Systems

S. H. Yang

Photoinduced Reactions in Complexes Consisting of a Magnesium Cation and Methyl Halide Molecules $\mathrm{Mg}^{+}\left(\mathrm{XCH}_{3}\right)_{n}$

Z. C. Ying

Near Field Optical Microscopy of Fractal Materials

I. A. Yu, Y.-C. Chen and C.-W. Lin

Quantum Interference in Ultracold Atoms

Accelerator Physics

Z. M. Dai G. M. Liu and N. Huang

Design of the SSRF Magnet Lattice

S. Fang and S. Fu

Preliminary Consideration of the ADS Research in China

Y. C. Huang

The Physics and Status of the Proposed NTHU Relativistic Photon-Electron Dynamics Laboratory

Y. Lin and D. Tong

The R\&D of Electron Linear Accelerators and their Applications in Tsinghua

University, Beijing

X. J. Wang

Future Light Source Technology R\&D at the BNL ATF

Y. Zhao

Power Compression by Means of Thyratron in Low Frequency

Program

Author Index 